\title{
Analyse von Preispolitiken mit Excel
}

Prof. Dr. Dr. h.c. Dieter Kirschke und Dr. Kurt Jechlitschka, Berlin

In diesem Beitrag möchten wir zeigen, wie Fragen der Preispolitik mit Excel modelliert und gelöst werden können. Durch diese Herangehensweise werden die theoretischen Grundlagen der Analyse von Preispolitiken geschärft und Modellierungstechniken vermittelt, die für eigene und weitergehende Fragestellungen genutzt werden können. Inhaltlich konzentriert sich der Beitrag auf die neoklassischen Grundlagen der Instrumenten- und Politikanalyse und der Allokationspolitik; methodische Grundlage ist ein partielles Marktmodell.

Prof. Dr. Dr. h.c. Dieter Kirschke ist Professor für Agrarpolitik an der Humboldt-Universität zu Berlin mit den Arbeitsschwerpunkten angewandte Wirtschaftspolitik und internationale Agrar-und Ernährungspolitik.

Dr. Kurt Jechlitschka ist Wissenschaftler am Fachgebiet Agrarpolitik mit dem Arbeitsschwerpunkt Markt- und Optimierungsmodelle in den Wirtschaftswissenschaften.

\section{Einleitung}

Bei der Analyse von Preispolitiken mit Excel geht es darum, theoretische Überlegungen und Modellierungsansätze in ein Tabellenkalkulationsprogramm zu übertragen und dieses für die Analyse zu nutzen. Methodisch gehen wir von einem bewusst „spartanisch“ gehaltenen partiellen Marktmodell aus, das für komplexere Fragestellungen ausgeweitet werden kann. Im folgenden Kapitel wird zunächst gezeigt, wie ein solches Modell in Excel formuliert werden kann. Zur Analyse verschiedener Fragen werden die „Mehrfachoperation“ und der „Solver“ genutzt. Im dritten Kapitel werden Modell und Analyse dann auf wohlfahrtsökonomische Fragen ausgeweitet. Im vierten Kapitel betrachten wir komplexere Fragen der Gestaltung von Preispolitiken und Trade offs zwischen Zielen. Der Beitrag schließt mit Überlegungen zur Nutzung von Excel für weitergehende Analysen von Preispolitiken ab.

Der Beitrag setzt Grundkenntnisse über Mikroökonomie und Wirtschaftspolitik, ökonomische Modellierung und die Nutzung von Tabellenkalkulationsprogrammen voraus. Er soll dazu anregen, 
die Nutzung solcher Programme für die Analyse von Preispolitiken selbst auszuprobieren. In unserer Darstellung orientieren wir uns an der Version Excel 97; die Beschreibungen sind aber ebenso gültig für jüngere Excel-Versionen. Zu beachten ist allerdings, dass aus der „Mehrfachoperation“ bei Excel 97 die Funktion Tabelle (unter „Daten“, „Tabelle“) bei den deutschen Versionen ab Excel 2000 geworden ist.

\section{Angebot, Nachfrage und Preispolitik}

Grundlage für die Analyse von Preispolitiken ist die Formulierung von Angebots- und Nachfragefunktionen und die Ableitung weiterer Variablen in einem Marktmodell. Dieses Modell ist dann in Excel zu übertragen.

\subsection{Ein Marktmodell}

Bei der Formulierung von Angebots- und Nachfragefunktionen wird oft unterstellt, dass diese Funktionen konstante Elastizitäten haben, also isoelastisch sind. Betrachten wir also solche isoelastischen Funktionen, die in Anlehnung an ihre „Erfinder“ in den Wirtschaftswissenschaften auch Cobb-Douglas-Funktionen genannt werden. Für die Angebotsfunktion gilt zunächst:

$$
\mathrm{q}^{\mathrm{s}}(\mathrm{p})=\mathrm{c} \cdot \mathrm{p}^{\varepsilon^{\mathrm{s}}} ; \varepsilon^{\mathrm{s}}>0
$$

mit $q^{s}(\cdot) \quad-\quad$ Angebotsfunktion

$\mathrm{q}^{\mathrm{s}} \quad$ - Angebotsmenge

$\mathrm{p} \quad$ - Inlandspreis

c - Angebotskonstante

$\varepsilon^{\mathrm{s}} \quad$ - Angebotselastizität.

Analog gilt für die Nachfragefunktion:

$$
q^{d}(p)=d \cdot p^{\varepsilon^{d}} ; \varepsilon^{d}<0
$$

mit $q^{d}(\cdot) \quad-\quad$ Nachfragefunktion

$q^{d} \quad-\quad$ Nachfragemenge

d - Nachfragekonstante

$\varepsilon^{\mathrm{d}} \quad$ - Nachfrageelastizität.

Stellen wir uns nun vor, dass ein Land den Inlandspreis p je nach politischer Zielvorstellung unabhängig vom Weltmarktpreis $\mathrm{p}^{\mathrm{w}}$ setzt. Eine solche Preispolitik kann durch eine direkte Preissetzung und Mengeninterventionen verwirklicht werden; in einem marktwirtschaftlichen System findet man jedoch meistens indirekte Formen der Preisbeeinflussung durch „Subventionierung“ bzw. „Besteuerung“ wirtschaftlicher Aktivitäten. Eine protektionistische Preispolitik $\left(\mathrm{p}>\mathrm{p}^{\mathrm{w}}\right)$ etwa lässt 
sich im Importfall durch eine Importbesteuerung, einen Zoll, umsetzen, im Exportfall durch eine Exportsubvention.

Unterstellen wir ferner, dass der Weltmarktpreis für das betrachtete Land exogen gegeben ist; es gilt also die „Kleines Land“-Annahme, nach der der Weltmarktpreis durch nationale Angebots- und Nachfrageänderungen nicht beeinflusst wird. Formal gilt demnach, dass $p$ und $p^{w}$ in unserem Modell exogene Variablen darstellen und weitere Variablen beeinflussen können. Für die Erlöse der Produzenten etwa gilt:

$$
E(p)=q^{s}(p) \cdot p \text {. }
$$

Die Ausgaben der Verbraucher ergeben sich wie folgt:

$$
A(p)=q^{d}(p) \cdot p .
$$

Devisen(einnahmen) lassen sich definieren als

$$
D\left(p, p^{w}\right)=\left(q^{s}(p)-q^{d}(p)\right) p^{w},
$$

und für die Budget(einnahmen) gilt:

$$
B\left(p, p^{w}\right)=\left(q^{d}(p)-q^{s}(p)\right)\left(p-p^{w}\right) .
$$

\subsection{Umsetzung des Modells in Excel}

Das skizzierte Marktmodell kann nunmehr in Excel formuliert werden.

Step 1 Geben Sie in B4 und C4 den Inlands- und den Weltmarktpreis von z.B. 10 ein. In B8:E8 sollen die Werte der Parameter $\mathrm{c}, \mathrm{d}, \varepsilon^{\mathrm{s}}$ und $\varepsilon^{\mathrm{d}}$ stehen. In Zelle B8 tragen wir für die Angebotskonstante c zunächst den Wert 1 ein, und für die Nachfragekonstante $d$ in Zelle C8 nehmen wir ebenfalls den Wert 1. Die Angebotselastizität von z.B. 0,3 tragen wir in D8 und die Nachfrageelastizität von $-0,4$ in E8 ein.

Step 2 In Zelle E4 lautet dann die Formel für die Angebotsmenge =B8*B4^D8. Die Nachfragefunktion in $\mathrm{F} 4$ erhalten wir entsprechend als $\quad=\mathrm{C} 8 * \mathrm{~B} 4{ }^{\wedge} \mathrm{E} 8$.

Step 3 Um die Werte für c und d zu bestimmen, müssen Sie Angebots- und Nachfragefunktion kalibrieren, d.h. es sind die Funktionen in (1) und (2) so anzupassen, dass sich für den festen Inlandspreis von $\mathrm{p}=10$ die gewünschten (oder am Markt beobachteten) Mengen für das Angebot von z.B. $q^{\mathrm{s}}=70$ und für die Nachfrage des Gutes von z.B. $q^{\mathrm{d}}=110$ ergeben. Damit erhalten wir: 


$$
\mathrm{c}=\frac{70}{10^{0,3}} \quad \text { bzw. } \quad \mathrm{d}=\frac{110}{10^{-0,4}} \text {. }
$$

Die entsprechenden Formeln in den Zellen B8 und C8 lauten damit $=70 / 10^{\wedge} \mathrm{D} 8 \mathrm{bzw}$. $=110 / 10^{\wedge} \mathrm{E} 8$.

Step 4 In H4 bis K4 tragen Sie entsprechend (3) - (6) die Formeln für die Erlöse $=\mathrm{E} 4 * \mathrm{~B} 4$, die Ausgaben $=\mathrm{F} 4 * \mathrm{~B})$, die Devisen $=(\mathrm{E} 4-\mathrm{F} 4) * \mathrm{C} 4$ und das Budget $=(\mathrm{F} 4-\mathrm{E} 4) *(\mathrm{~B} 4-\mathrm{C} 4)$ ein und fertig ist Ihr Marktmodell, das Sie durch geeignete Bezeichnungen in der dritten Zeile ergänzen sollten (siehe Abb. 1).

\begin{tabular}{|c|c|c|c|c|c|c|c|c|c|c|c|}
\hline \multicolumn{12}{|c|}{ Microsoft Excel - Abb1.xls } \\
\hline \multicolumn{3}{|c|}{88} & \multicolumn{2}{|c|}{$==70 / 10^{\wedge} \mathrm{D} 8$} & & \multirow[b]{2}{*}{$\mathrm{F}$} & \multirow[b]{2}{*}{ G } & \multirow[b]{2}{*}{$\mathrm{H}$} & \multirow[b]{2}{*}{ I } & \multirow[b]{2}{*}{ J } & \multirow[b]{2}{*}{ K } \\
\hline & A & B & C & $\mathrm{D}$ & $E$ & & & & & & \\
\hline 1 & & \multicolumn{4}{|c|}{ Cobb-Douglas-Marktmodell } & & & & & & \\
\hline 2 & & & & & & & & & & & \\
\hline 3 & & $p$ & $p^{\prime \prime \prime}$ & & Angebot & Nachfrage & & Erlöse & Ausgaben & Devisen & Budget \\
\hline 4 & & 10 & 10 & & 70 & 110 & & 700 & 1100 & -400 & 0 \\
\hline 5 & & & & & & & & & & & \\
\hline 6 & \multicolumn{3}{|c|}{ Konstanten: } & \multicolumn{2}{|c|}{ Preiselastizität } & & & & & & \\
\hline 7 & & c & $d$ & \multicolumn{2}{|c|}{ des Angebots der Nachfrage } & & & & & & \\
\hline 8 & & 35,083106 & 276,30751 & 0,3 & $-0,4$ & & & & & & \\
\hline
\end{tabular}

Abb. 1 Cobb-Douglas-Marktmodell

Die Werte in Ihrem Modell beschreiben die Situation bei Freihandel und für $p^{\mathrm{w}}=10$. Um die Konsequenzen einer protektionistischen Preispolitik bestimmen zu können, setzen Sie einfach den Inlandspreis auf z.B. 15 oder 25. Sie erhalten dann die Werte der definierten Variablen für eine Importsituation und eine Exportsituation.

\subsection{Mehrfachoperation und Solver}

Um die Wirkung von Preispolitiken besser erfassen zu können, ist die grafische Darstellung relevanter Größen als Funktion der betrachteten Preise hilfreich. Eine Frage wäre etwa, wie sich Devisen und Budget für $10 \leq \mathrm{p} \leq 25$ entwickeln.

Step 5 Zur Beantwortung dieser Frage verfahren Sie wie folgt: Tragen Sie in G11 die Zahl 10 ein und gehen Sie wieder auf Zelle G11. Unter „Bearbeiten“, „Ausfüllen“ und „Reihe“ wählen Sie die Option „Reihe in Spalten“ und tragen den „Endwert“ 25 ein. In H9 schreiben Sie die Formel =J3 und kopieren Sie H9 in die Felder H9:I10. Jetzt markieren Sie G10:I26 und wählen „Daten“, „Mehrfachoperation“; klicken Sie in das Feld „Werte aus Spalte“ und dann auf B4. So erhalten Sie die Werte für die Devisen und das Budget für die Preise von 10 bis 25. Markieren Sie nun G9:I26 und wählen Sie im 
Diagrammassistenten z.B. den Diagrammtyp „Linie mit Datenpunkten“. Nach einigem Editieren erhalten Sie so das Diagramm, wie in Abb. 2 angegeben. 


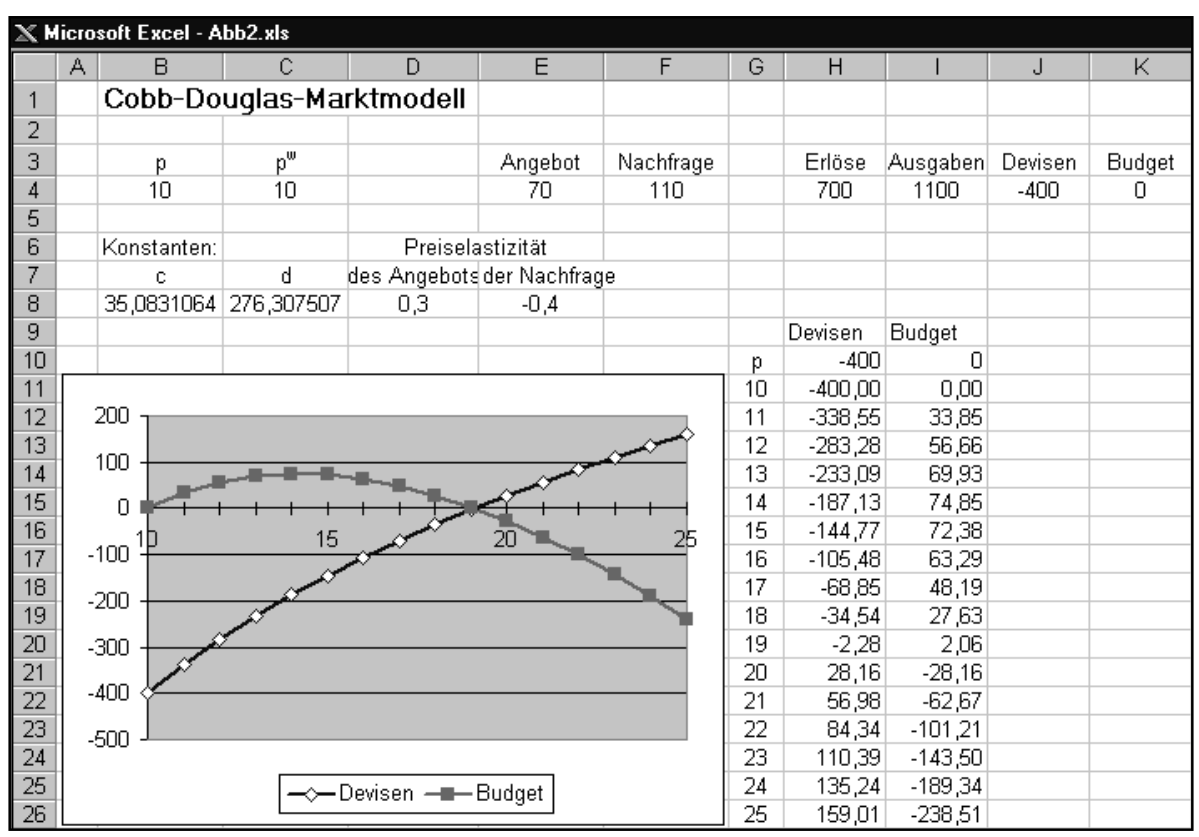

Abb. 2 Devisen und Budget als Funktion des Inlandspreises bei einem Weltmarktpreis von 10

Der Verlauf der Devisen- und Budgetfunktion macht deutlich, dass der Autarkiepreis bei $\mathrm{p} \approx 19$ liegt. Die streng konkave Budgetfunktion hat ihre Nullstellen offensichtlich bei Freihandel $p=p^{w}$ und bei Autarkie. Bei einem zwischen dem Weltmarktpreis $\mathrm{p}^{\mathrm{w}}$ und dem Autarkiepreis liegenden Inlandspreis entstehen Zoll- und somit Budgeteinnahmen mit einem Maximum bei $p \approx 14$, und bei einem über den Autarkiepreis hinausgehenden Inlandspreis entstehen wachsende Budgetausgaben durch Exportsubventionen. Die Devisen andererseits sind eine monoton steigende Funktion des Inlandspreises p mit einer Nullstelle beim Autarkiepreis.

Um den Autarkiepreis zu berechnen, kann der Solver genutzt werden.

Step 6 Dazu klicken Sie auf „Extras“ und „Solver...“. Ist der Menüpunkt „Solver...“ nicht vorhanden, so muss er über „Extras“, „Add-In-Manager“" aktiviert werden bzw. der Solver sogar mit der Original-CD nachinstalliert werden. Da wir zur Bestimmung des Autarkiepreises eigentlich keine Optimierungsaufgabe haben, ist die Wahl der Zielzelle gleichgültig, es muss aber eine Zelle mit einer Formel sein (z.B. E4), oder wir wählen einfach keine Zielzelle aus. Als veränderbare Zelle wählen Sie den Inlandspreis (durch Eintragen von B4 in die entsprechende Zeile oder durch Klicken auf dieses Feld und dann auf Zelle B4). Als Nebenbedingung fügen Sie E4 = F4 hinzu (zeigen oder eintragen). Damit haben wir den Solver eigentlich gesetzt (siehe Abb. 3 und 4). 


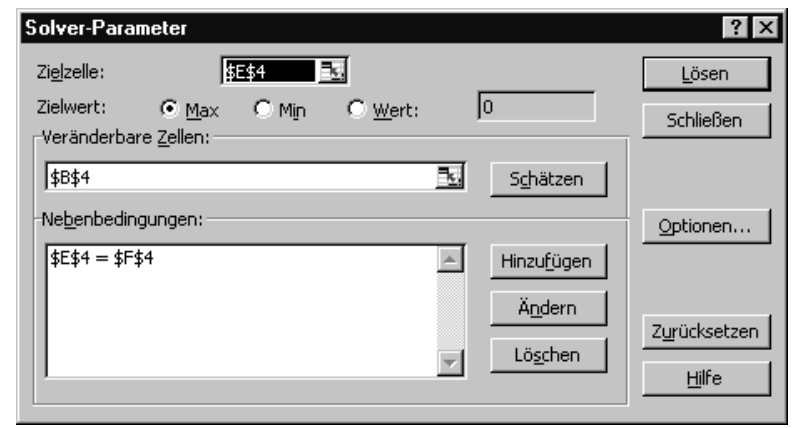

Abb. 3 Solver-Dialogbox

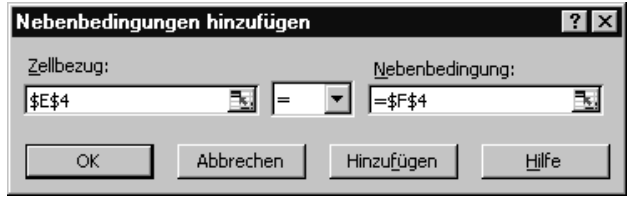

Abb. 4 Dialogbox zum Hinzufügen von Nebenbedingungen

Da unser Modell nicht linear ist, darf das Kästchen unter „Optionen...“, „Lineares Modell voraussetzen“ nicht aktiviert sein. Ebenfalls soll die Nicht-Negativität der Variablen (Preis) vorausgesetzt werden (siehe Abb. 5). Wählen Sie „OK“ und „Lösen“. Wir erhalten den Autarkiepreis von 19,07 und die Gleichgewichtsmenge von 84,96.

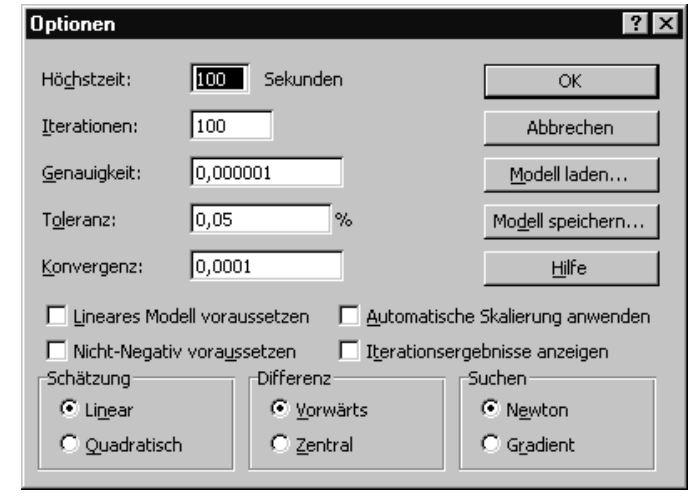

Abb. 5 „Optionen“-Dialogbox des Solvers

\section{Wohlfahrt und Verteilung}

Wohlfahrts- und Verteilungswirkungen spielen eine wesentliche Rolle bei der Analyse von Preispolitiken. Es gilt, relevante Wohlfahrtsvariablen $\mathrm{zu}$ formulieren und diese in die Analyse von Preispolitiken mit Excel zu integrieren. 


\subsection{Formulierung von Wohlfahrtsvariablen}

Nach dem Konzept der angewandten Wohlfahrtsökonomik wird die (maximale) Zahlungsbereitschaft als Wohlfahrtsindikator eines Konsumenten interpretiert. Diese Zahlungsbereitschaft, aggregiert für alle Konsumenten, wird als Fläche unter der Nachfragekurve bis zur nachgefragten Menge beschrieben. Wir sprechen auch vom Nutzen aus der Konsumaktivität, für den gilt:

$$
N(p)=\int_{0}^{q^{d}(p)} \widetilde{p}^{d}(v) d v
$$

$$
\begin{array}{llll}
\text { mit } & \mathrm{N} & - & \text { Nutzen } \\
& \widetilde{\mathrm{p}}^{\mathrm{d}}(\cdot) & - & \text { inverse Nachfragefunktion } \\
& \mathrm{v} & - & \text { Integrationsvariable, hier } \mathrm{q}^{\mathrm{d}} .
\end{array}
$$

Nach dem Konzept stellen die variablen Kosten in der Produktion Nutzenentgang, also Opportunitätskosten, dar, und diese werden als Fläche unter der Angebotsmenge bis zur angebotenen Menge ausgewiesen:

$$
\mathrm{K}(\mathrm{p})=\int_{0}^{\mathrm{q}^{\mathrm{s}}(\mathrm{p})} \widetilde{\mathrm{p}}^{\mathrm{s}}(\mathrm{v}) \mathrm{dv}
$$

$$
\begin{array}{clll}
\text { mit } & \mathrm{K} & - & \text { Kosten } \\
& \widetilde{\mathrm{p}}^{\mathrm{s}}(\cdot) & - & \text { inverse Angebotsfunktion } \\
\mathrm{V} & - & \text { Integrationsvariable, hier } \mathrm{q}^{\mathrm{s}} .
\end{array}
$$

In einer offenen Volkswirtschaft müssen schließlich Devisen als „wohlfahrtswirksam“ berücksichtigt werden. Deviseneinnahmen aus Exporten sind wohlfahrtserhöhend, weil sie einen Güteranspruch gegenüber dem Ausland und damit potentielle Bedürfnisbefriedigung darstellen. Devisenausgaben für Importe bedeuten demgegenüber, dass der Güteranspruch gegenüber dem Ausland sinkt; sie sind also wohlfahrtsmindernd. Fasst man diese Wohlfahrtskomponenten zusammen, so lässt sich folgende Wohlfahrtsfunktion formulieren:

$$
\mathrm{W}\left(\mathrm{p}, \mathrm{p}^{\mathrm{w}}\right)=\mathrm{N}(\mathrm{p})-\mathrm{K}(\mathrm{p})+\mathrm{D}\left(\mathrm{p}, \mathrm{p}^{\mathrm{w}}\right) .
$$

Analog kann man das Wohlfahrtsniveau auch als Summe der Wohlfahrt der Konsumenten (Konsumentenrente), der Wohlfahrt der Produzenten (Produzentenrente) und des Budgets formulieren. Die Konsumentenrente wird als Differenz der Fläche unter der Nachfragekurve bis zur nachgefragten Menge und der Ausgaben ausgewiesen; die Produzentenrente entspricht der Differenz aus den Erlösen und der Fläche unter der Angebotskurve bis zur angebotenen Menge; Budgeteinnahmen schließlich erhöhen potentiell die Konsummöglichkeiten von Mitgliedern einer Gesellschaft, etwa durch erhöhte Transferzahlungen, durch die Senkung von Steuern oder auch durch die Bereitstel- 
lung öffentlicher Güter, während Budgetausgaben eine Minderung gesellschaftlicher Konsummöglichkeiten darstellen.

\subsection{Integration der Wohlfahrtsvariablen in Excel}

Die beschriebenen Wohlfahrtsindikatoren sind nunmehr in Excel zu formulieren.

Step 7 Schaffen wir zunächst etwas Platz für die Kosten-, Nutzen- und Wohlfahrtsfunktion, die wir in die Felder H4 bis J4 eintragen. Dazu verschieben Sie den Bereich mit dem Funktionenblock H3:K4 nach K3:N4 (z.B.: H3:K4 markieren, den Mauszeiger über den Rahmen führen, der dann zum Pfeil wird, mit gedrückter linker Maustaste den neuen Bereich suchen und die Maustaste loslassen).

Step 8 Beginnen wir mit der Kostenfunktion, die wir im Feld H4 eintragen. Entsprechend (1) und (8) gilt für diese Funktion:

$$
\mathrm{K}=\mathrm{p} \cdot \mathrm{q}^{\mathrm{s}}(\mathrm{p})-\frac{1}{\varepsilon^{\mathrm{s}}+1} \mathrm{c} \mathrm{p}^{\varepsilon^{\mathrm{s}}+1}
$$

Tragen Sie also in Zelle H4 die Excel-Formel $=\mathrm{B} 4 * \mathrm{E} 4-\mathrm{B} 8 * \mathrm{~B} 4^{\wedge}(\mathrm{D} 8+1) /(\mathrm{D} 8+1)$ ein.

Step 9 Bei der Nutzenfunktion haben wir das Problem, dass die entsprechende Fläche unter der (inversen) Cobb-Douglas-Nachfragefunktion unendlich groß wäre, da das Integral den Wert $\infty$ hat. Wir ziehen uns aus der Affäre, indem wir das so ,auslaufende Integral“ bei einem entsprechend hohen Preiswert, dem „Quasi-Schnittpunkt“ der Nachfragekurve mit der Preisachse, und konkret bei $\mathrm{p}^{\mathrm{d}_{\mathrm{o}}}=100$ abschneiden. Dieses Vorgehen scheint akzeptabel, da viele Fragestellungen bei Marktmodellen auf die Änderungen der Zielwerte (hier des Nutzens) gerichtet sind und sich hierfür durch die Wahl der Obergrenze keine Probleme ergeben. Bei der Interpretation des Niveaus des Nutzens und von Zielvariablen, die auf der Nutzenvariable beruhen, wie Konsumentenrente und auch Wohlfahrt, ist natürlich Vorsicht geboten.

Entsprechend (2) und (7) gilt also:

$$
\mathrm{N}=\mathrm{p} \cdot \mathrm{q}^{\mathrm{d}}(\mathrm{p})+\frac{\mathrm{d}}{\varepsilon^{\mathrm{d}}+1}\left(100^{\varepsilon^{\mathrm{d}}+1}-\mathrm{p}^{\varepsilon^{\mathrm{d}}+1}\right)
$$

Entsprechend tragen Sie in Zelle I4 die Excel-Formel $=\mathrm{B} 4 * \mathrm{~F} 4+\mathrm{C} 8 /(\mathrm{E} 8+1)^{*}\left(100^{\wedge}(\mathrm{E} 8+1)-\mathrm{B} 4^{\wedge}(\mathrm{E} 8+1)\right)$ ein.

Step 10 Nun ist die Wohlfahrtsformel als Nutzen - Kosten + Devisen in J4 ein Kinderspiel. Ebenso einfach gestalten sich die Formeln für die Produzenten- und Konsumentenrente 
in O4 und P4 als Differenz von Erlösen und Kosten bzw. als Differenz von Nutzen und Ausgaben. Vergleichen Sie die Ergebnisse Ihres Modells mit den Werten in Abb. 6.

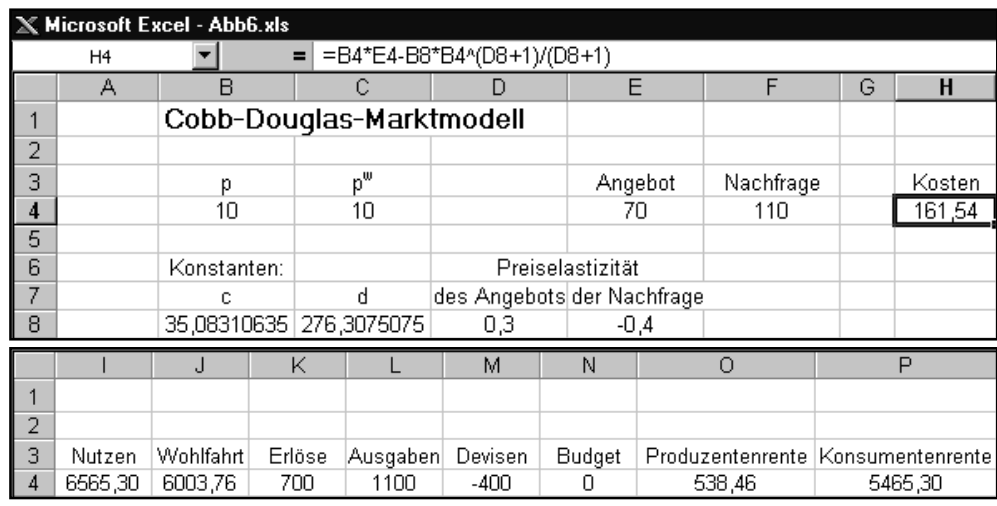

Abb. 6 Cobb-Douglas-Marktmodell mit Wohlfahrtsindikatoren

\subsection{Wohlfahrtsanalysen}

Auf der Grundlage des formulierten Modells kann man nun beispielsweise zeigen, dass Freihandel gemäß des Theorems des komparativen Vorteils zur Maximierung der Wohlfahrt führt.

Step 11 Gehen Sie z.B. von einem Inlandspreis 15 als Startwert und dem Weltmarktpreis 10 aus. Setzen Sie den Solver wie folgt: Zielzelle: J4, die Wohlfahrt soll maximiert werden; Zielwert: Max; Veränderbare Zelle: B4; Nebenbedingungen: Keine. Als Ergebnis erhalten Sie den wohlfahrtsmaximierenden Inlandspreis von 10, also den Weltmarktpreis. Das Ergebnis ist allerdings nur ein Näherungswert. Da die Wohlfahrtsfunktion keine lineare Funktion der Preise ist, ist auch das Optimierungsmodell kein lineares, und der Solver bietet eine von den Einstellungen unter „Optionen“ abhängige, vom exakten Wert 10 eventuell leicht abweichende Näherungslösung des genutzten Iterationsverfahrens an. Arbeiten Sie auch mit anderen Startwerten für den Inlandspreis und vergleichen Sie die vom Solver ermittelten Lösungen!

Für verschiedene Inlandspreise kann man die Konsequenzen für Wohlfahrt und Verteilung unmittelbar ablesen. Mit der Mehrfachoperation kann man solche Wohlfahrts- und Verteilungseffekte gegenüber Freihandel grafisch darstellen, z.B. für $10 \leq p \leq 20$.

Step 12 Tragen Sie zunächst als Ausgangswerte den Inlandspreis von 10 und den Weltmarktpreis von 10 ein. Verfahren Sie dann wie folgt: Tragen Sie in G11 die Zahl 10 ein und gehen Sie wieder auf Zelle G11. Unter „Bearbeiten“, „Ausfüllen“ und „Reihe“ wählen Sie die Option „Reihe in Spalten“ und tragen den „Endwert“ 20 ein. In H9 schreiben Sie die Formel $=\mathrm{O} 3$ und kopieren Sie H9 in die Zellen H9:I10. In J9 schreiben Sie die 
Formel $=\mathrm{J} 3$ und in $\mathrm{J} 10$ die Formel $=\mathrm{J} 4$. Jetzt markieren Sie G10:J21 und wählen „Daten“, „Mehrfachoperation“, klicken Sie in das Feld „Werte aus Spalte“ und dann auf B4. So erhalten Sie die Werte für die Produzenten- und Konsumentenrente und die Wohlfahrt bei den ganzzahligen Inlandspreisen zwischen 10 und 20 und können diese Funktionen wieder (wie im Step 5) über den Diagrammassistenten darstellen.

Step 13 Um die Abweichungen der jeweiligen Werte von den Freihandelswerten zu erhalten, tragen Sie in Zelle H26 die Formel $=$ H11-\$H\$11 ein und kopieren diese in den Bereich H26:H36; in Zelle I26 und J26 verfahren Sie mit den Formeln =I11-\$I\$11 bzw. $=\mathrm{J} 11-\$ \mathrm{~J} \$ 11$ analog. Den Vergleich mit den Freihandelswerten realisieren wir dabei durch die Verwendung von absoluten Bezügen mit Hilfe des \$-Zeichens.

Haben Sie in G26:G36 die Reihe 10 bis 20 und in H25:J25 geeignete Bezeichnungen stehen, so können Sie über die Markierung von G25:J36 hier ebenfalls eine gewünschte Grafik mit dem Diagrammassistenten erzeugen. Vergleichen Sie Ihre Ergebnisse mit Abb. 7.

Die Abbildung macht deutlich, dass die Wohlfahrtsverluste und die Umverteilungen zu Gunsten der Produzenten und zu Lasten der Konsumenten mit wachsendem Inlandspreis progressiv zunehmen. Diese zunehmenden Wohlfahrtsverluste machen die „Kosten“ einer protektionistischen Preispolitik in Bezug auf diese Zielvariable deutlich. Beachten Sie auch die Flexibilität des Modells und der grafischen Darstellungen bezüglich einer Änderung des Weltmarktpreises.

Die Wohlfahrtsgewinne des Freihandels gegenüber Autarkie, die ,gains from trade“, sind andererseits um so größer, je mehr das Freihandelsgleichgewicht vom Autarkiegleichgewicht abweicht. Entsprechend lässt sich eine ,gains from trade“-Kurve bestimmen, die das Wohlfahrtsniveau bei Freihandel bei unterschiedlichen Weltmarktpreisen angibt. 


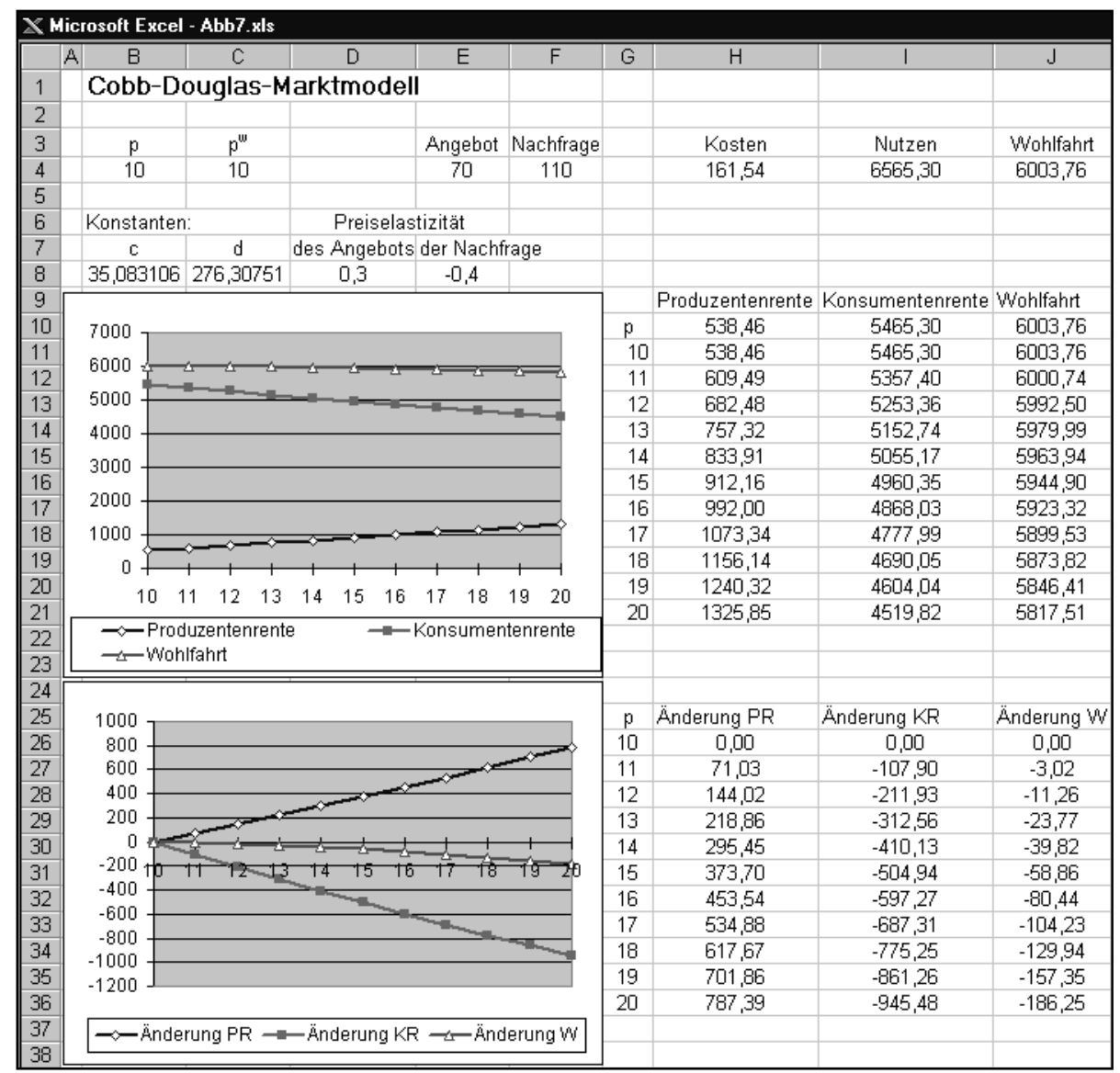

Abb. 7 Wohlfahrt, Konsumentenrente und Produzentenrente als Funktion des Inlandspreises bei einem Weltmarktpreis von 10

Step 14 Zur Ermittlung dieser Kurve tragen Sie in C4 die Formel =B4 ein und verfahren Sie wie unter Step 12, indem Sie als Datenreihe etwa 10 bis 30 verwenden und die Wohlfahrtsfunktion darstellen. Vergleichen Sie Ihr Ergebnis mit Abb. 8. 


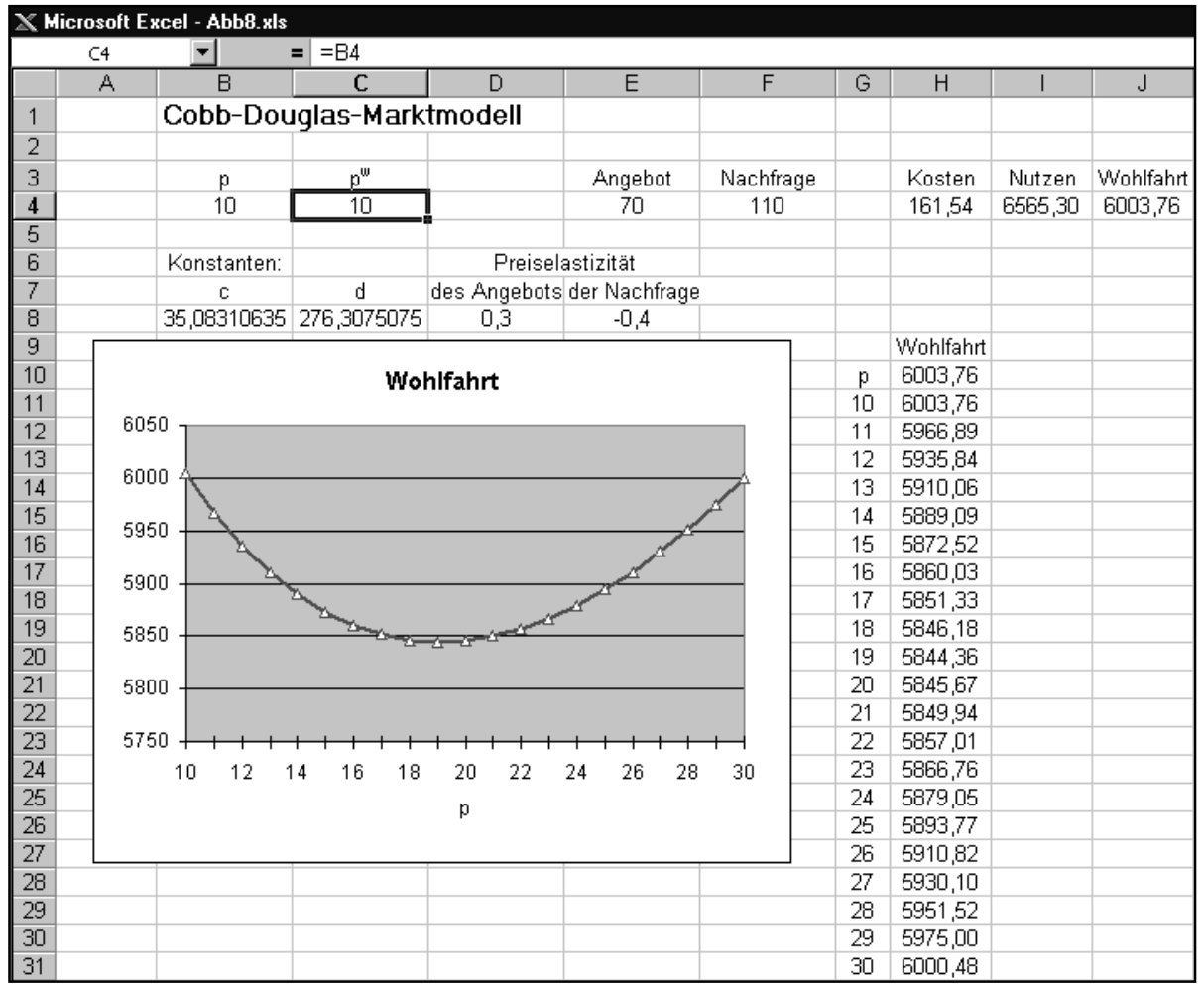

Abb. 8 „Gains from trade“-Kurve

\section{Gestaltung von Preispolitiken}

Wir hatten gezeigt, dass in unserem einfachen Modell eine Politik, bei der der Inlandspreis dem Weltmarktpreis entspricht, also Freihandel, zu einer Maximierung der Wohlfahrt führt. Aus gesamtwirtschaftlicher Sicht erscheint eine solche Politikgestaltung durchaus vernünftig; tatsächlich ist die Praxis der Gestaltung von Preispolitiken oft vielschichtiger und komplexer. Mit Excel können die Konsequenzen unterschiedlicher Gestaltungsansätze untersucht und Trade offs zwischen Zielen aufgezeigt werden.

\subsection{Optimierungsfragen}

Bei der Gestaltung von Preispolitiken ist die Betrachtung nur einzelner Komponenten der formulierten Wohlfahrtsfunktion $\mathrm{zu}$ Lasten anderer verbreitet. Mit einer protektionistischen Preispolitik etwa könnten im Importfall gegenüber Freihandel Budgeteinnahmen erzielt werden. Ein für eine solche Politik optimaler, d.h. budgetmaximierender Inlandspreis müsste zwischen dem Weltmarkt- und dem Autarkiepreis liegen, wie in Abb. 2 gezeigt. Auch eine Anhebung des Inlandspreises zur Erhöhung der Devisen ist oft diskutiert worden, als Importsubstitutionspolitik im Importfall oder als Exportförderungspolitik im Exportfall. Für eine solche Politik kann es keinen optimalen, hier: devisenmaximierenden Preis geben; denn jede Anhebung des Inlandspreises führt 
zu einer Erhöhung der Devisen. Ein wirkliches Optimierungsproblem entsteht also erst, falls Beschränkungen zu berücksichtigen sind, etwa Budgetausgaben oder Wohlfahrtsverluste.

Optimale Inlandspreise bei unterschiedlicher Zielsetzung können leicht mit dem Solver bestimmt werden.

Step 15 Soll das Budget maximiert werden, so ist der Solver wie folgt zu setzen: Zielzelle: N4; veränderbare Zelle: B4. Als Ergebnis erhalten Sie den optimalen Inlandspreis p = 14,15. Maximieren wir andererseits die Devisen (Zielzelle: M4) unter der Budgetrestriktion (Nebenbedingung: N4 >=-100 bzw. -200), so liegt der optimale Inlandspreis bei $b \approx 22$ bzw. $\mathrm{p} \approx 24$. Die Budgetrestriktion begrenzt also die Exportsubventionen in einer Überschusssituation (vgl. Abb. 9).

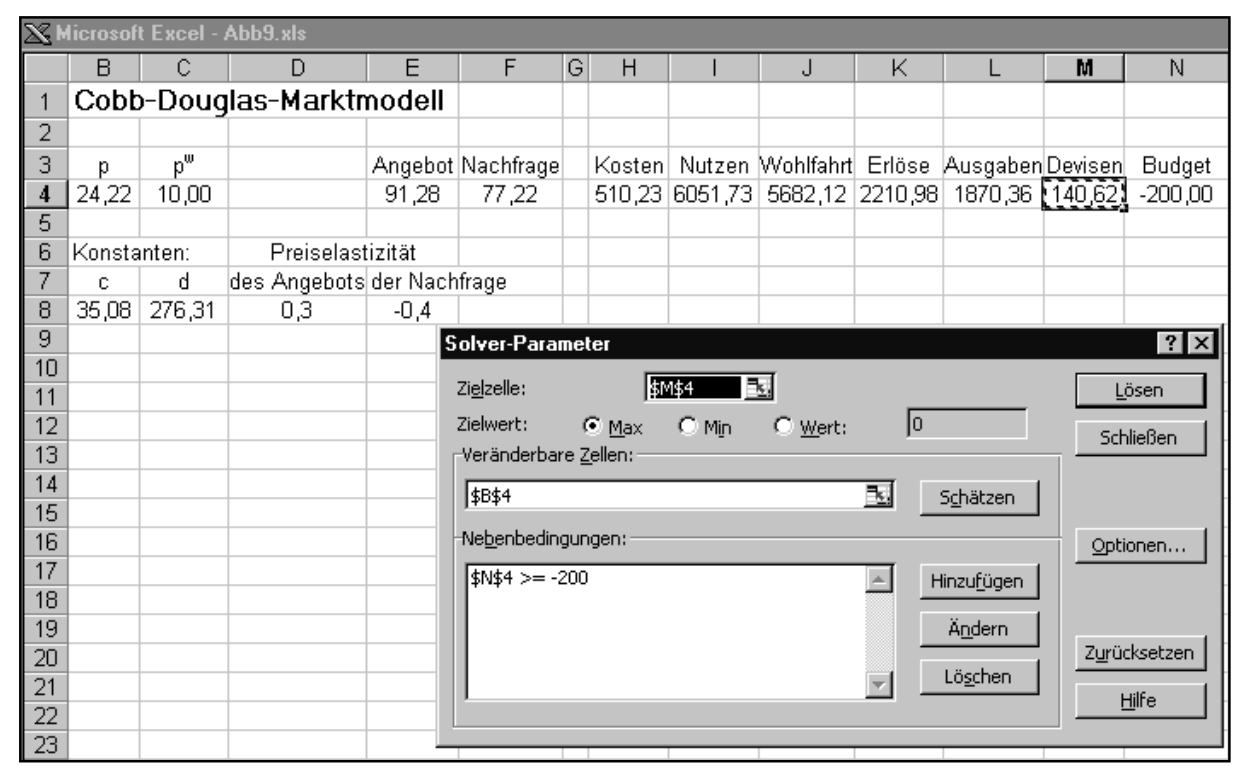

Abb. 9

Maximierung der Devisen bei einer Beschränkung der Budgetausgaben auf 200

\subsection{Trade offs zwischen Zielen}

Eine Kurve maximaler Zielverwirklichung zwischen verschiedenen Zielen, eine Trade off-Kurve, zeigt verschiedene Optionen für die Gestaltung von Preispolitiken auf. Eine solche Trade off-Kurve, etwa zwischen Produzentenrente und Budget, gibt an, welcher maximale Wert der Produzentenrente bei einem bestimmten Budgetniveau realisiert werden kann oder auch umgekehrt. In diesem Beispiel ist freilich zu beachten, dass die Konsumentenrente als Element der Wohlfahrt schlichtweg nicht berücksichtigt wird.

Step 16 Will man für die Gestaltung der Preispolitik die Trade off-Kurve zwischen Produzentenrente und Budget etwa bis zu einer Höhe der Budgetausgaben bis 400 bestimmen, so kann wieder der Solver wie in Step 15 genutzt werden, jedoch für alle 
Budgetschranken zwischen 0, -50, -100 bis -400. Dazu erzeugen wir im Bereich G14:G22 die entsprechenden Zahlenwerte und tragen in H14:H22 die von den SolverLösungen abhängigen Werte der Produzentenrente ein. Wir müssen also jeweils die rechte Seite unserer Nebenbedingungen (vgl. Abb. 9) ändern.

Markieren Sie nun die beiden Datenreihen und generieren Sie mit dem Diagrammassistenten (Typ Punkt (XY) mit interpolierter Linie) eine gewünschte Trade off-Kurve (s. Abb. 10 und 11). Unter „Diagramm-Optionen...“ können Sie auch den Titel und die Bezeichnung der Achsen gestalten.

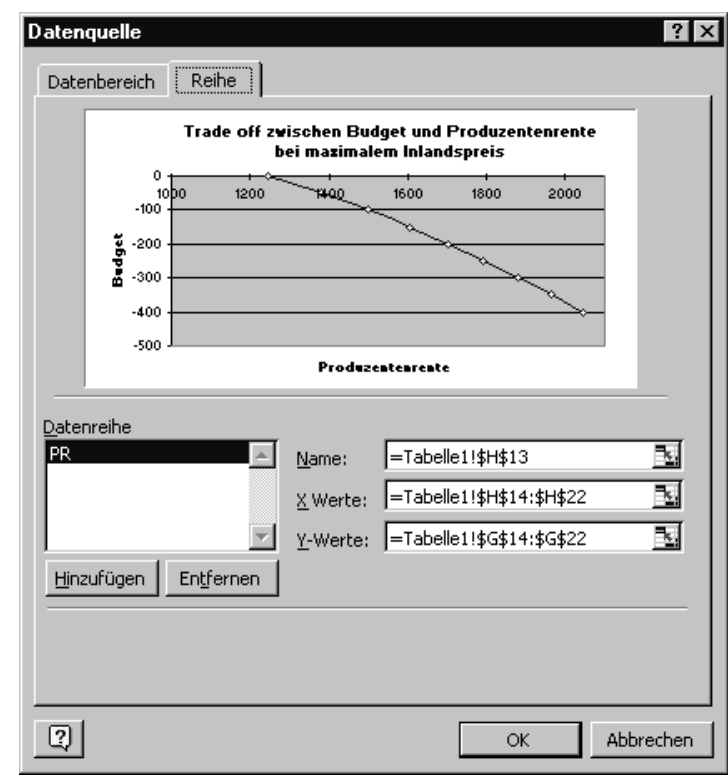

Abb. 10 Dialogbox „Datenquelle“, „Reihe“ 


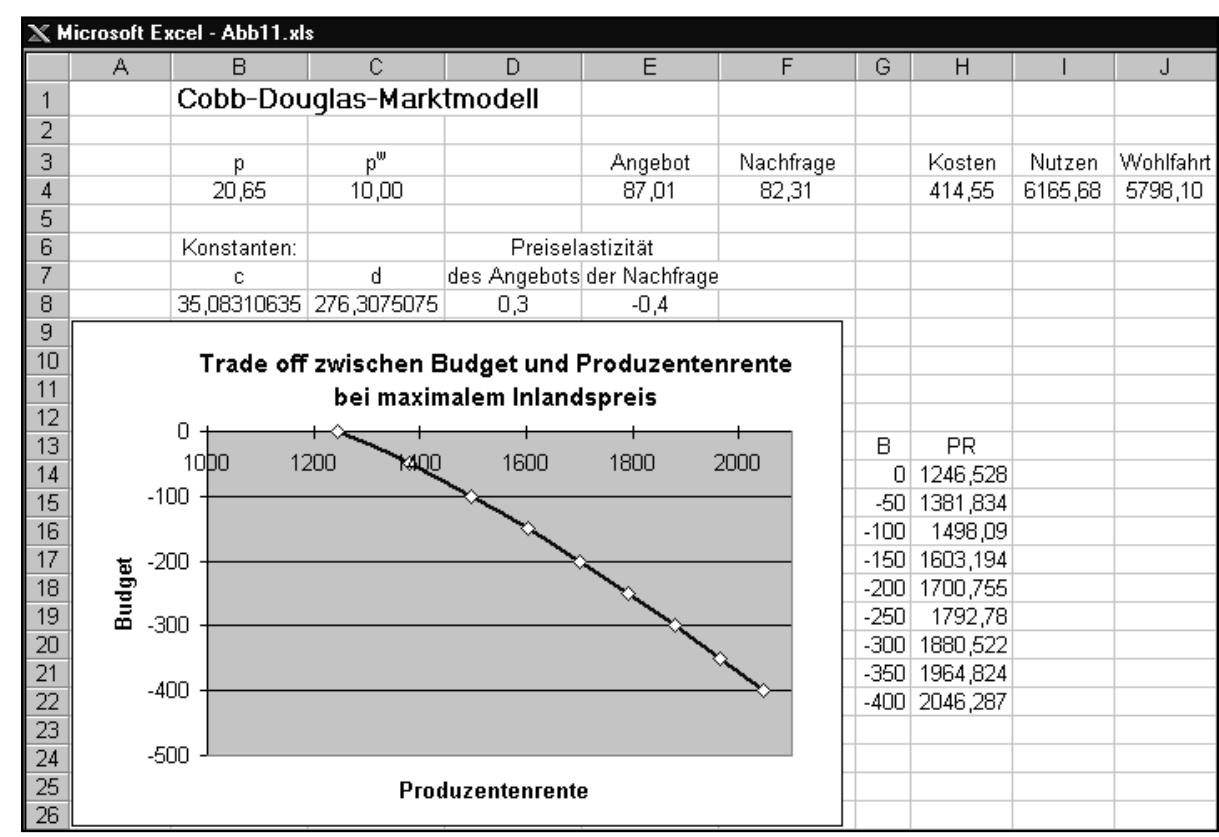

Abb. 11

Trade off zwischen Budget und Produzentenrente bei maximalem Inlandspreis

\section{Weiterführende Fragen}

Die Analyse von Preispolitiken mit Excel lässt sich in einfacher Weise und in vielerlei Hinsicht ausbauen. So können preispolitische Instrumente wie eine Protektionsrate oder auch eine Angebotsund Nachfragesubvention (bzw. -steuer) explizit modelliert werden. Das Marktmodell kann ebenso leicht um externe Effekte ergänzt und für verschiedene Regionen formuliert werden, um etwa die Konsequenzen von Preispolitiken in integrierten Märkten wie der Europäischen Union zu untersuchen oder die Konsequenzen nationaler Preispolitiken für Drittländer aufzuzeigen, wenn man die „Kleines Land-Annahme“ aufgibt. Mehrmarktmodelle schließlich erlauben eine weitere Ausweitung der Analyse.

Kirschke und Jechlitschka (2002) zeigen auf, wie solche weiterführenden Fragen mit Excel umgesetzt werden können. Die Analyse von Preispolitiken mit Excel stellt sich als ein wirkungsvoller Ansatz dar, die theoretischen Grundlagen zu schärfen und angewandte Probleme eigenständig zu modellieren und zu lösen. 


\section{Literatur}

Eine ausführliche Darstellung zur Nutzung von Excel für angewandte Fragen der Mikroökonomie und Wirtschaftspolitik findet sich bei:

Kirschke, D., K. Jechlitschka, Angewandte Mikroökonomie und Wirtschaftspolitik mit Excel, München 2002.

Zur Nutzung von Excel in den Wirtschaftswissenschaften und insbesondere in der Betriebswirtschaftslehre siehe auch:

Winston, W.L., Financial Models Using Simulation and Optimization, Newfield, USA 1999.

Winston,W.L., S.C. Albright, Practical Management Science, 2. Aufl., Wadsworth 2001.

$\mathrm{Zu}$ den theoretischen Grundlagen und zur Modellierung mikroökonomischer Fragen siehe:

Chiang, A.C., Fundamental Methods of Mathematical Economics, 3. Aufl., Singapore 1984.

Fritsch, M., T. Wein, H.-J. Ewers, Marktversagen und Wirtschaftspolitik : Mikroökonomische Grundlagen staatlichen Handelns, 4. erw. Aufl., München 2001.

Mas-Colell, A., M.D. Whinston, J.A. Green, Microeconomic Theory, New York, Oxford 1995.

Microsoft, Originalhandbücher und Online-Hilfe zu Excel 97 bzw. Excel 2000.

Pindyck, R.S., D.L. Rubinfeld, Mikroökonomie, 4. Aufl., München, Wien 1998.

Powell, S.G., Leading the Spreadsheet Revolution, in: ORMS Today 24, Vol. 6 (1997), S. 114-125.

Schumann, J., U. Meyer, W. Ströbele, Grundzüge der mikroökonomischen Theorie, 7. neubearb. und erw. Aufl., Berlin 1999.

Varian, H.R., Grundzüge der Mikroökonomik, 5. überarb. Aufl., München, Wien 2001. 